\title{
A false positive FDG PET/CT result in a patient with prosthetic heart valve disease
}

\author{
(1) Petra Grubić Rotkvić', \\ DZrinka Planinić ${ }^{*}$, \\ (-Ines Zadro Kordić', \\ DOgnjen Čančarević1, \\ 이ea Friščić1, \\ DAnte Pašalić', \\ (1D)Jozica Šikić1,2
}

'University Hospital "Sveti Duh", Zagreb, Croatia ${ }^{2}$ University of Zagreb, School of Medicine, Zagreb, Croatia
KEYWORDS: infective endocarditis, Duke criteria, prosthetic valve, positron emission tomography. CITATION: Cardiol Croat. 2021;16(9-10):303. | https://doi.org/10.15836/ccar2021.303

*ADDRESS FOR CORRESPONDENCE: Zrinka Planinić, Klinička bolnica "Sveti Duh“, Sv. Duh 64, HR-10000 Zagreb, Croatia. / Phone: +385-91-3714-308 / E-mail: zrinkaplaninic@gmail.com

ORCID: Petra Grubić Rotkvić, https://orcid.org/0000-0002-2587-1932 • Zrinka Planinić, https://orcid.org/0000-0001-8664-3338 Ines Zadro Kordić, https://orcid.org/0000-0002-0754-7194 • Ognjen Čančarević, https://orcid.org/0000-0002-1285-8042 Tea Friščić, https://orcid.org/0000-0003-3189-8661 • Ante Pašalić, https://orcid.org/0000-0001-5989-6495 Jozica Šikić, https://orcid.org/0000-0003-4488-0559

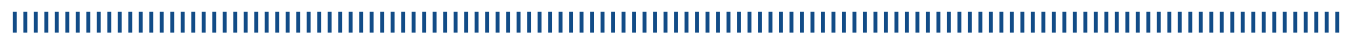

Introduction: Prosthetic valve endocarditis (PVE) represents 10-30\% of all cases of endocarditis, occurring in $1-6 \%$ of patients with prosthetic valves. Diagnosis of PVE is made according to the modified Duke criteria that are less sensitive than for native valve endocarditis (NVE) given limitation of echocardiography ${ }^{1}$. Even though ${ }^{18} \mathrm{~F}$-fluorodeoxyglucose positron emission tomography/computed tomography (FDG-PET/CT) is a more accurate diagnostic tool for PVE included as a major criterion in the

European Society of Cardiology (ESC) Guidelines for the management of infective endocarditis (IE), increased periprosthetic FDG uptake not always reflects infection ${ }^{2}$

Case report: 75 -year-old male patient with a prior history of Bentall procedure in 2013 has been hospitalized for fever of unknown origin. Blood cultures came positive on Streptococcus pluranimalium and a high suspicion of PVE was raised. Transesophageal echocardiography (TEE) showed a suspected vegetation on mechanical aortic valve without compromising valve function (Figure 1). No other signs of septic embolization were found, except subconjunctival hemmorrhage. Since two major and two minor Duke criteria for definite IE were met, the patient was started on standard antibiotic treatment according to ESC guidelines. Despite completed targeted antibiotic treatment, the patient remained subfebrile without clear clinical manifestation of infection with repeated negative blood cultures and no signs of vegetation on TEE. FDG PET/CT was performed and showed diffuse perivalvular FDG uptake pattern consistent with inflammation. While awaiting for the PET/CT result, the patient became afebrile with no laboratory or clinical signs of infection. In consultation with cardiac surgeon a strategy of watchful waiting was taken. The patient remained stable during one year follow-up.
FIGURE 1. Transesophageal echocardiography showing suspected vegetation on an mechanical aortic valve.
RECEIVED:

July 24, 2021

ACCEPTED:

August 5, 2021

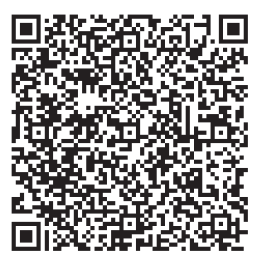

Conclusion: Homogenous FDG uptake may be present in the perivalvular area in the absence of PVE even years after valve implantation ${ }^{1}$. Since the factors used to distinguish normal and abnormal FDG uptake patterns are not yet standardized ${ }^{2}$, clinical judgement and multidisciplinary treatment approach are still crucial in decision making.

LITERATURE IIIIIIIIIIIIIIIIIIIIIIIIIIIIIIIIIIIIIIIIIIIIIIIIIIIIIIIIIIIIIIIIIIIIIIIIIIIIIIIIIIIIIIIIIIIIIIIII

1. Mathieu C, Mikail' N, Benali K, lung B, Duval X, Nataf P, et al. Characterization of 18F-Fluorodeoxyglucose Uptake Pattern in Noninfected Prosthetic Heart Valves. Circ Cardiovasc Imaging. 2017 Mar;10(3):e005585. https://doi.org/10.1161/CIRCIMAGING.116.005585

2. Erba PA, Slart RHJA. Pattern recognition on fluorodeoxyglucose positron emission tomography/computed tomography in infective endocarditis: within the normal limits? Eur Heart J Cardiovasc Imaging. 2020 Jan 1;21(1):34-35. https://doi.org/10.1093/ehjci/jez254 